\title{
Closed Loop System Identification with Genetic Algorithms
}

\author{
Mark S. Whorton* \\ NASA/Marshall Space Flight Center, Huntsville, Alabama 35812
}

\begin{abstract}
High performance control design for a flexible space structure is challenging since high fidelity plant models are difficult to obtain a priori. Uncertainty in the control design models typically require a very robust, low performance control design which must be tuned on-orbit to achieve the required performance. Closed loop system identification is often required to obtain a multivariable open loop plant model based on closed-loop response data. In order to provide an accurate initial plant model to guarantee convergence for standard local optimization methods, this paper presents a global parameter optimization method using genetic algorithms. A minimal representation of the state space dynamics is employed to mitigate the non-uniqueness and over-parameterization of general state space realizations. This control-relevant system identification procedure stresses the joint nature of the system identification and control design problem by seeking to obtain a model that minimizes the difference between the predicted and actual closed-loop performance.
\end{abstract}

\section{Introduction}

Implicit in the design of high performance control systems is the availability of an accurate model of the system to be controlled. System identification methods are typically directed toward obtaining accurate nominal models of an open loop system with bounds on the associated uncertainty. Recognizing this dependence on accurate nominal design models, robust control theory has been developed to accommodate modeling errors. Recently attention has been drawn to the fact that the issues of system identification and control design must be treated as mutually dependent. ${ }^{1}$ Since the magnitude and spectrum of excitation forces are controller dependent, an appropriate model for control design cannot be determined independent of the controller. Since the model that is most appropriate depends on the control design, the open-loop response of a model is not sufficient to indicate the fidelity of the model for control design. A better approach from a closed loop performance perspective is closed-loop system identification, that is the identification of the open-loop plant given closed-loop response data and knowledge of the compensator dynamics.

An approach to iterative closed-loop system identification and controller redesign is given by Liu and Skelton where a state space model is estimated using the q-Markov Cover algorithm and a controller is designed using the Output Variance Constraint algorithm. ${ }^{2}$ q-Markov Cover theory describes all realizations of a linear system which match the first q Markov parameters and covariance parameters of the true system generated by pulse responses. First the entire closed-loop system is estimated and then using knowledge of the compensator dynamics, the plant is extracted. However, the identified open-loop plant has dimension equal to the controller dimension plus the dimension of the identified closed-loop plant. Model order reduction is used to remove the superfluous states. A similar approach is to formulate a method which first obtains the closed-loop Markov parameters and then the open-loop Markov parameters are recovered. ${ }^{3}$ A discrete time state space realization is then obtained from the Markov parameters.

A classical approach to parameter estimation which has been extended to closed-loop system identification is the prediction error approach. ${ }^{4}$ This optimization method estimates the parameters of a linear system by minimizing the squared sum of the errors between the actual measurements and the predicted measurements. Recent work extended the prediction error method to closed-loop identification for multivariable systems. ${ }^{5}$ Building on a method developed for estimation of the parameters of an open-loop system in canonical form

*TD54/Guidance, Navigation, and Control Systems Group, Associate Fellow AIAA. 
from open-loop data, a new procedure for closed-loop system identification was developed and demonstrated therein. As with all local optimization methods that utilize inverted Hessians and gradients for searching, the optimization method was found to be severely limited by the accuracy of the initial guess. In this paper, a global optimization step utilizing genetic algorithms is introduced to determine a coarse optimal solution to initialize the local optimization process previously developed. ${ }^{5}$

\section{Closed Loop System Identification}

The traditional approach to control design is to obtain a nominal model of the open loop plant, $\hat{P}$, which is the basis for control design. Since $\hat{P}$ is an approximation of the true plant, $P$, the model based compensator, $C_{\hat{P}}$, must provide a certain level of robust stability (i.e., $C_{\hat{P}}$ must internally stabilize $\hat{P}$ and $P$ ). In the system identification process an attempt is made to bound the model error $\|P-\hat{P}\|$, which determines the amount of robustness required by the control design. It is also desirable that the controller provide some level of performance robustness; that is the achievable performance should not differ significantly from the nominal design performance.

An upper bound on achievable performance is ${ }^{6}$

$$
\begin{aligned}
& \left\|J\left(P, C_{\hat{P}}\right)\right\| \leq \\
& \left\|J\left(\hat{P}, C_{\hat{P}}\right)\right\|+\left\|J\left(P, C_{\hat{P}}\right)-J\left(\hat{P}, C_{\hat{P}}\right)\right\|
\end{aligned}
$$

where

$\left\|J\left(P, C_{\hat{P}}\right)\right\|:$ the achieved performance

$\left\|J\left(\hat{P}, C_{\hat{P}}\right)\right\|:$ the nominal performance, and

$\left\|J\left(P, C_{\hat{P}}\right)-J\left(\hat{P}, C_{\hat{P}}\right)\right\|:$ the performance differential.

The choice of a specific performance metric $J$ and norm $\|*\|$ is determined by the control design methodology.

High performance control design for a flexible space structure is especially challenging since high fidelity nominal plant models are difficult to obtain. To achieve high performance requires:

- high nominal performance $\left(\Leftrightarrow\left\|J\left(\hat{P}, C_{\hat{P}}\right)\right\|\right.$ small $)$

- robust performance

$$
\left(\Leftrightarrow\left\|J\left(P, C_{\hat{P}}\right)-J\left(\hat{P}, C_{\hat{P}}\right)\right\| \ll\left\|J\left(\hat{P}, C_{\hat{P}}\right)\right\|\right)
$$

The second condition is actually a requirement on model fidelity and indicates that the nominal closed-loop system model must closely approximate the actual closed-loop system performance when the compensator $C_{\hat{P}}$ is used. Therefore, the fitness of the nominal model is a function of the compensator and must be judged from a closed-loop perspective. Interestingly, good fitness is not guaranteed by good open-loop model matching nor is it precluded by poor open-loop model matching. ${ }^{1}$

The closed-loop system identification process may be considered as one step of an iterative closed-loop system identification and control design procedure. It is assumed that a moderately accurate dynamic model of the system to be controlled is available for the initial low authority controller design. However, this initial design model is not of sufficient fidelity to permit high authority control design. The objective of the closedloop system identification procedure is to refine the initial control design model based on closed-loop response data.

In the development of a closed-loop system identification method, consideration must be given to the non-uniqueness of the state space realization of the system dynamics. Although there are an infinite number of equivalent state space realizations for a system, a system with $n$ states, $n u$ inputs, and $n y$ outputs can be uniquely expressed with a minimum of $n(n u+n y)$ parameters. In order to circumvent the non-uniqueness problem, this paper will implement an approach which realizes the open-loop system matrices in a unique, minimal form and directly identifies the canonical parameters from closed-loop response data.

Proper selection of the objectives of system identification and control design further stresses the joint nature of the identification and control problem. The objective of the closed-loop identification procedure employed herein is to obtain a model $\hat{P}$ that minimizes the performance differential $\|T-\hat{T}\|_{2}$ where $T$ is the actual closed-loop system and $\hat{T}$ is the identified closed-loop system. This system norm cannot be 
evaluated since $T$ is not known. However the actual and predicted closed-loop measurements are known and an equivalent objective is to minimize the prediction error of the closed-loop system, $\|y-\hat{y}\|_{2}$. The actual and predicted closed-loop system outputs, $y$ and $\hat{y}$, respectively, are determined for the same set of inputs. The least squares cost functional for control dependent closed-loop system identification then is

$$
J=\frac{1}{2} \int_{0}^{t_{f}}(y-\hat{y})^{T} W(y-\hat{y}) d t
$$

where $W$ is a constant matrix chosen to weight the relative importance of different measurement outputs. The control objective is matched with the identification objective by designing the controller to minimize the $H_{2}$ criterion $\|\hat{T}\|_{2}$.

\section{State Space Canonical Realization}

A state space representation of the open loop plant may be given by:

$$
\begin{aligned}
\dot{z} & =\mathcal{F} z+\mathcal{G} u, \quad z(0)=z_{0} \\
\hat{y} & =\mathcal{H} z
\end{aligned}
$$

The objective is to identify some $\mathcal{F}, \mathcal{G}, \mathcal{H}$, and $z_{0}$ which represents the dynamics of the unknown system based on knowledge of the inputs, $u(t)$, and noisy measurements, $y(t)$. An estimate of the unknown parameters may be obtained by minimizing the cost functional given in Eq. 2. Directly minimizing $J$ for the general state space realization results in a nonlinear optimization process, but in the absence of measurement noise, a linear formulation may be obtained. Recognizing that for a perfect model the output in Eq. 4 will exactly equal the measurements and $y-\hat{y}=0$, this difference can be fed back to the model with arbitrary gains $\mathcal{K}$ and $\mathcal{M}$ according to

$$
\begin{aligned}
\dot{z} & =\mathcal{F} z+\mathcal{G} u+\mathcal{K}(y-\mathcal{H} z) \\
\hat{y} & =\mathcal{H} z+\mathcal{M}(y-\mathcal{H} z)
\end{aligned}
$$

which can also be written

$$
\begin{aligned}
\dot{z} & =\mathcal{F}_{N} z+\mathcal{G}_{N} u+\delta \mathcal{G} u+\mathcal{K} y \\
z(0) & =z_{N 0}+\delta z_{0} \\
\hat{y} & =\mathcal{H}_{N} z+\mathcal{M} y
\end{aligned}
$$

by use of the definitions

$$
\begin{aligned}
\mathcal{F}_{N} & =\mathcal{F}-\mathcal{K H} \\
\mathcal{G}_{N} & =\mathcal{G}-\delta \mathcal{G} \\
\mathcal{H}_{N} & =(I-\mathcal{M}) \mathcal{H} \\
z_{N 0} & =z_{0}-\delta z_{0}
\end{aligned}
$$

The elements of $(\mathcal{F}-\mathcal{K} \mathcal{H})$ and $(I-\mathcal{M}) \mathcal{H}$ may be chosen independently of the unknown elements of $\mathcal{F}$ and $\mathcal{H}$ by using a maximum of $n * n y$ parameters in $\mathcal{K}$ and $\mathcal{M}$ when the structure of the system is in a specific form. As a consequence, $\mathcal{F}_{N}, \mathcal{G}_{N}, \mathcal{H}_{N}$, and $z_{N 0}$ are chosen and the unknown parameters are contained in $\mathcal{K}, \mathcal{M}, \delta \mathcal{G}$, and $\delta z_{0}$. The vector of uncertain parameters to be estimated is

$$
\gamma=\left[\begin{array}{c}
\operatorname{vec}(\mathcal{K}) \\
\operatorname{vec}(\delta \mathcal{G}) \\
\operatorname{vec}(\mathcal{M}) \\
\operatorname{vec}\left(\delta z_{0}\right)
\end{array}\right]
$$

A canonical realization of the system dynamics is necessary so that the unknown parameters in $\mathcal{F}$ are coefficients of measured states. This paper utilizes the observer canonical form. ${ }^{7}$ It can be shown that if the plant dimension is an even multiple of the number of outputs and the first $n$ rows of the observability matrix are linearly independent, then the realization is canonical and $\mathcal{H}$ will consist only of ones and zeros. Otherwise, some elements of $\mathcal{H}$ will be included as unknown parameters in the estimation procedure. 


\section{A. Closed-Loop System Realization}

A similar approach may be taken for identification of the closed-loop system parameters by expressing the plant in observer canonical form and exploiting the structure of the closed-loop matrices. For the plant given by

$$
\begin{aligned}
\dot{x} & =A x+B_{1} w+B_{2} u \\
y & =C_{2} x
\end{aligned}
$$

and a dynamic compensator

$$
\begin{aligned}
\dot{x}_{c} & =A_{c} x_{c}+B_{c} y \\
u & =-C_{c} x_{c}
\end{aligned}
$$

the resulting closed-loop dynamics are

$$
\begin{aligned}
{\left[\begin{array}{c}
\dot{x} \\
\dot{x}_{c}
\end{array}\right]=} & {\left[\begin{array}{cc}
A & -B_{2} C_{c} \\
B_{c} C_{2} & A_{c}
\end{array}\right]\left[\begin{array}{c}
x \\
x_{c}
\end{array}\right] } \\
& +\left[\begin{array}{cc}
B_{1} & B_{2} \\
0 & 0
\end{array}\right]\left[\begin{array}{l}
w \\
u
\end{array}\right] \\
y= & {\left[\begin{array}{cc}
C_{2} & 0 \\
0 & I
\end{array}\right]\left[\begin{array}{c}
x \\
x_{c}
\end{array}\right] }
\end{aligned}
$$

If the plant $\left(A, B_{2}, C_{2}\right)$ matrices are expressed in observer form, then the $C_{2}$ matrix consists of ones and zeros and the unknown parameters in $A$ are coefficients of the measured transformed states. (As stated earlier, in some cases the transformation may not be canonical resulting in the $C_{2}$ matrix having additional nonzero elements, but these parameters can be estimated as well.) Comparison of Eqs. 3 - 4 with Eqs. 19 20 indicates that

$$
\begin{gathered}
\mathcal{F}=\left[\begin{array}{cc}
A & -B_{2} C_{c} \\
B_{c} C_{2} & A_{c}
\end{array}\right] \\
\mathcal{G}=\left[\begin{array}{cc}
B_{1} & B_{2} \\
0 & 0
\end{array}\right] \\
\mathcal{H}=\left[\begin{array}{cc}
C_{2} & 0 \\
0 & I
\end{array}\right]
\end{gathered}
$$

Since the only unknowns in $\mathcal{F}$ and $\mathcal{G}$ are the plant matrices $A, B_{1}$, and $B_{2}$, the unknown parameter matrices are defined as

$$
\begin{aligned}
\mathcal{K} & =\left[\begin{array}{cc}
\mathcal{K}_{11} & -\mathcal{K}_{12} C_{c} \\
0 & 0
\end{array}\right] \\
\delta \mathcal{G} & =\left[\begin{array}{cc}
\delta \mathcal{G}_{11} & \delta \mathcal{G}_{12} \\
0 & 0
\end{array}\right]
\end{aligned}
$$

In the case of a true canonical form for the plant, $\mathcal{H}$ is completely known. Compatibility of $\mathcal{K}$ and $\mathcal{H}$ with $\mathcal{F}$ requires $\mathcal{H}$ to have the form given by Eq. 23 which implies that the compensator state vector must be included in the measurement vector used in the identification process. Note that $\mathcal{K}_{11}$ corresponds to the unknown parameters in $A, \mathcal{K}_{12}$ corresponds to the unknown parameters in $B_{2}, \delta \mathcal{G}_{11}$ corresponds to the unknown parameters in $B_{1}$, and $\delta \mathcal{G}_{12}=\mathcal{K}_{12}$. Hence for closed-loop system identification, the unknown parameter vector becomes:

$$
\gamma=\left[\begin{array}{c}
\operatorname{vec}\left(\mathcal{K}_{11}\right) \\
\operatorname{vec}\left(\mathcal{K}_{12}\right) \\
\operatorname{vec}\left(\delta \mathcal{G}_{11}\right) \\
\operatorname{vec}\left(\delta z_{0}\right)
\end{array}\right]
$$


Local optimization methods have been shown to be quite effective for parameter estimation when a good initial estimate is available. ${ }^{5}$ However in the absence of good initial estimates, these methods tend to be unsuitable due to numerical sensitivity. Local optimization methods often require the inversion of a large data matrix which tends to be ill-conditioned. Ensuring full-rank of the data matrix requires sufficiently rich excitation in all of the modes to be identified which is often quite difficult in practice due to limitations such as sensor and actuator dynamics. Methods which do not involve matrix inversion such as genetic algorithms have potential for the closed-loop parameter estimation. An additional benefit of genetic algorithms is that the system identification can be operating in the background as part of an autonomous identification/controller tuning process. Hence this paper presents a genetic algorithm for the initial (coarse, global) estimation step with the objective of providing a sufficiently accurate initial guess for the final (local) optimization step using the equation error method.

\section{Genetic Algorithms}

Scientists and engineers often borrow concepts from nature to motivate and inform the design process. One such innovation is the computational field of genetic algorithms. Genetic algorithms have seen increased attention over the last two decades in a wide range of engineering applications from aircraft design to operations research. Genetic algorithms are mathematical methods for global search and optimization that are based on the mechanics of natural selection and the science of genetics. ${ }^{8}$

In nature, natural selection operates on biological systems to determine the fittest species for a given ecological niche. The fittest species tend to survive and propagate the genetic information that rendered the species most fit. In this sense, natural selection has a stabilizing effect that tends to increase the presence of the fittest species through subsequent generations by selecting the fittest members for propagation. Mating within the population also tends to mix the genetic information of the population such that beneficial traits of parents can be combined to improve the fitness of the offspring. Hence the operations of selection and reproduction tend to preserve the fittest members of a species and in some instances improve the fitness of individuals within the population through genetic mixing. However selection and reproduction both operate on a fixed gene pool with fixed genetic information. Truly innovative biological designs require the introduction of new biological information.

The only known natural mechanism for introducing new biological information is mutation, which essentially modifies the genetic code of a species. Mutations are random, relatively rare, and almost always harmful to the species. However the exceptional beneficial mutation introduces new genetic information to the gene pool which may lead to more fit members to be selected and reproduced. This paradigm of biological optimization has been fruitfully applied to engineering optimization methods in the general field of evolutionary computation and genetic algorithms in particular.

At the cellular level, biological information is coded in one or more chromosomes which serve to describe the function and construction of the biological system. Chromosomes are comprised of strings of DNA which can be divided into substrings called genes. Each gene is a functional block of DNA that encodes the biochemical workhorse of the cell - protein molecules. The genetic code is simply a mapping from DNA strings to individual proteins and the genome is an organism's set of genes that comprises the set of biological characteristics or traits of the organism. So a trait (such as hair color) is encoded by a gene and the different possible realizations of that trait (such as brown, black, etc.) are called alleles.

To carry over the biological paradigm as a computational paradigm, the chromosome may be thought of as a candidate solution to the optimization problem. The chromosome is a string formed by the concatenation of individual genes, or parameters to be optimized. Alleles correspond to the values that may be taken by the parameter (either one or zero in a binary representation, a real number in a floating point representation).

Genetic algorithms consist of coding a population of candidate solutions and then operating on the population to determine a new population for the next generation. Several generations are typically iterated upon until the population converges to its most fit set of solutions. The operators for one generation typically include selection, recombination, and mutation.

Selection implements the operation of natural selection by evaluating the "fitness" of each member (from the cost functional to be optimized) and giving more copies of the better solutions in the population for the next generation. Selection takes many forms such as roulette wheel selection, where the probability of an individual being propagated to the next generation is weighted by the fitness; tournament selection, where the random members compete "head to head;" and truncation, where the top "n" members are selected and 
copied multiple times to fill the population for the next generation.

Recombination implements biological reproduction by combining two parents to form two children for the next generation. Two strings chosen at random are cut at a random location in a gene (for binary encoded strings) or chromosome (for floating point encoded strings) and the remaining portions of the strings are swapped. Also known as crossover, this step serves to randomly shuffle the information in the gene pool, but it does not introduce new information in the gene pool. For example, if every member in a population has a " 1 " in the $i^{t h}$ string location, then neither reproduction or recombination will produce a "0" in that location.

The introduction of new information is accomplished through the third operator, mutation. Mutation in a genetic algorithm is accomplished by randomly altering an allele. For a binary encoding, mutation simply flips a bit at random locations as a function of the probability of mutation. For a floating point encoded string, mutation perturbs a random allele is some fashion such as a random variation over some range of parameter variation. Taken together, these three operators serve to advance the population from generation to generation in a manner that tends to improve the fitness of either the population as a whole or the best members of the population.

The next section presents an example application of genetic algorithms to the problem of parameter estimation for closed loop system identification.

\section{System Identification Implementation}

Standard local optimization methods typically involve differentiation of an objective function and inversion of matrices that may tend toward ill-conditioning. Genetic algorithms are much simpler to implement than standard methods because genetic algorithms merely evaluate the objective function. This distinction also implies that genetic algorithms are better suited for global searches since gradients inherently provide only local topological information. Clearly the over-parameterization of a general state-space representation will introduce difficulties for the genetic algorithm search just as it does for standard optimization methods. Implementing the canonical realization for the closed loop system identification mitigates this difficulty and permits the genetic algorithm to search for the best set of unknown parameters for the minimal realization. Hence the objective of the genetic algorithm for closed loop system identification is to determine the plant parameters which minimizes the error between the predicted and measured outputs as quantified by the objective function in Eq. 2.

Given an initial closed loop plant described by $\mathcal{F}_{0}, \mathcal{G}_{0}$, and $\mathcal{H}_{0}$, the updated plant matrix $\mathcal{F}_{\text {new }}$ may be given by

$$
\mathcal{F}_{\text {new }}=\mathcal{F}_{0}-\Delta \mathcal{F}
$$

Since the unknown plant parameters to be estimated are elements of $\left(A, B_{1}, B_{2}\right)$, inspection of Eqs. 21 and 10 indicates that

$$
\Delta \mathcal{F}=\left[\begin{array}{cc}
\Delta A & -\Delta B_{2} C_{c} \\
0 & 0
\end{array}\right]=\mathcal{K H}
$$

With $\mathcal{K}$ and $\mathcal{H}$ compatibly partitioned as defined in Eqs. 24 and 23, Eq. 28 yields $\Delta A=\mathcal{K}_{11} C_{2}$, or

$$
\mathcal{K}_{11}=\Delta A C_{2}^{+}
$$

where the right inverse (pseudo-inverse) of $C_{2}$ is given by

$$
C_{2}^{+}=C_{2}^{T}\left(C_{2} C_{2}^{T}\right)^{-1}
$$

The variation in $A$ can be treated as a multiplicative percent variation from the initial matrix $A_{0}$ by the definition

$$
\Delta A=\Delta_{A} A_{0}
$$

where $\Delta_{A}$ is the matrix

$$
\Delta_{A}=\operatorname{diag}\left[\delta A_{i}\right], \quad \forall i=1: n * p
$$

and the "diag" notation indicates a diagonal matrix with the elements in brackets along the diagonal. Each $\delta A_{i}$ represents the percent uncertainty in the $i^{t h}$ uncertain element of the open loop $A$ matrix. Using the canonical realization in this manner allows us to isolate the individual uncertain elements of the open loop 
plant matrices from within the closed loop realizations as linear multiplicative coefficients. Likewise, Eq. 28 yields

$$
\Delta B_{2} C_{c}=\mathcal{K}_{12} C_{c} \mathcal{H}_{22}
$$

For this case where the realization is canonical, $\mathcal{H}_{22}$ is the identity matrix and hence

$$
\mathcal{K}_{12}=\Delta B_{2}=\Delta_{B_{2}} B_{2,0}
$$

where $B_{2,0}$ is the initial value of $B_{2}$ and $\Delta_{B_{2}}$ is a diagonal matrix of percent variations analogous to Eq. 32 . Finally, the updated $\mathcal{G}$ plant matrix is given by Eq. 11 which by direct inspection yields

$$
\delta G_{11}=\Delta B_{1}=\Delta_{B_{1}} B_{1,0}
$$

where $B_{1,0}$ is the initial value of $B_{1}$ and $\Delta_{B_{1}}$ is a diagonal matrix of percent variations analogous to Eq. 32 .

This representation allows the chromosome to be a concatenation of the percent variations of the individual uncertain plant parameters. Beginning with a population of chromosomes, each binary chromosome string is decomposed into substrings, or genes, that represent the percent variation of each plant parameter. The substrings are decoded into floating point numbers that are the diagonal elements of $\Delta_{A}, \Delta_{B_{1}}$, and $\Delta_{B_{2}}$. These floating point numbers are then used in Eqs. $29-35$ to form the parameter vector $\gamma$ defined by Eq. 26 (neglecting uncertainty in the initial conditions $\delta z_{0}$ ). Hence each chromosome in the population is mapped to a corresponding $\gamma$ from which the closed loop plant matrices are formed. A time response of the corresponding closed loop plant is compared to the measured time response of the true system and the fitness of each chromosome in the population is finally determined by Eq. 2.

\section{Application Example}

The four disk structural system ${ }^{9}$ is an excellent example of a flexible space structure and is used herein as a test case for closed loop system identification using genetic algorithms. The four disk model can be configured with a rigid body mode and coupled nonminimum phase flexible modes (as a consequence of noncolocated sensors and actuators). As illustrated in Figure 1, four disks are rigidly attached to a flexible

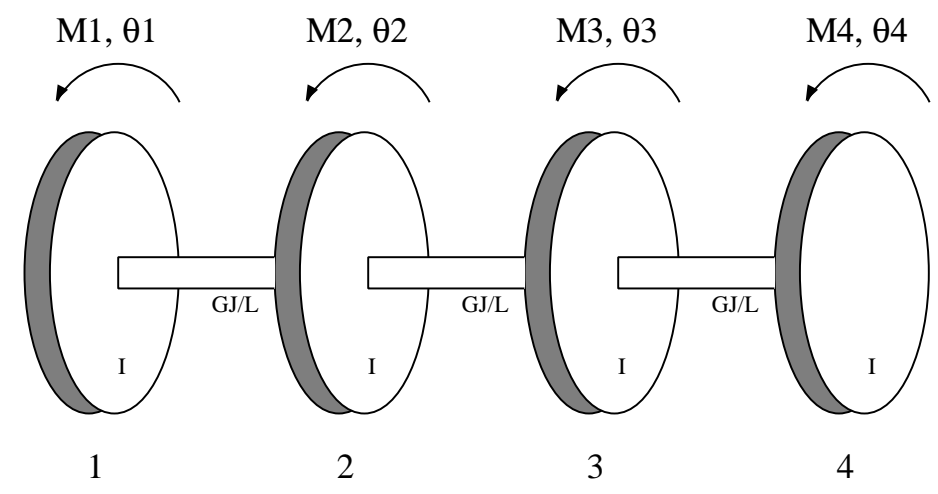

Figure 1. Four Disk System

axial shaft with control torque applied to selected disks and the angular displacement of selected disks measured. The equations of motion may be written as

$$
\begin{aligned}
I_{1} \ddot{\theta}_{1}+K_{1}\left(\theta_{1}-\theta_{2}\right) & =M_{1} \\
I_{2} \ddot{\theta}_{2}+K_{2}\left(\theta_{2}-\theta_{3}\right)-K_{1}\left(\theta_{1}-\theta_{2}\right) & =M_{2} \\
I_{3} \ddot{\theta}_{3}+K_{3}\left(\theta_{3}-\theta_{4}\right)-K_{2}\left(\theta_{2}-\theta_{3}\right) & =M_{3} \\
I_{4} \ddot{\theta}_{4}-K_{3}\left(\theta_{3}-\theta_{4}\right) & =M_{4}
\end{aligned}
$$

or

$$
\bar{M} \ddot{q}+\bar{K} q=\bar{B} u
$$


where the mass matrix is

$$
\bar{M}=\left[\begin{array}{cccc}
I_{1} & 0 & 0 & 0 \\
0 & I_{2} & 0 & 0 \\
0 & 0 & I_{3} & 0 \\
0 & 0 & 0 & I_{4}
\end{array}\right]
$$

and the stiffness matrix with $(G J / L)_{i}=K_{i}$ is given by

$$
\bar{K}=\left[\begin{array}{cccc}
K_{1} & -K_{2} & 0 & 0 \\
-K_{1} & K_{1}+K_{2} & -K_{2} & 0 \\
0 & -K_{2} & K_{2}+K_{3} & -K_{3} \\
0 & 0 & -K_{3} & K_{3}
\end{array}\right]
$$

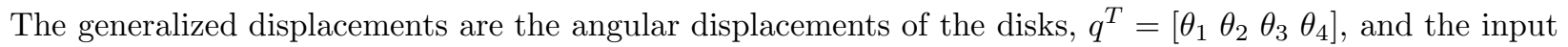
vector consists of the moments applied to each disk, $u^{T}=\left[\begin{array}{llll}M_{1} & M_{2} & M_{3} & M_{4}\end{array}\right]$. Defining the state vector as $x^{T}=\left[\begin{array}{ll}q^{T} & \dot{q}^{T}\end{array}\right]$ results in the state space formulation

$$
\dot{x}=A x+B_{2} u
$$

where

$$
A=\left[\begin{array}{cc}
0 & I \\
\bar{M}^{-1} \bar{K} & 0
\end{array}\right] \quad B_{2}=\left[\begin{array}{c}
0 \\
\bar{M}^{-1} \bar{B}
\end{array}\right]
$$

Note that Eq. 19 includes an explicit input coefficient matrix, $B_{1}$, for the disturbance input, $w$. This disturbance input typically consists of noise or uncertainties at the plant input and is used as a design parameter for controller synthesis. In the control design context, the $B_{1}$ matrix includes the control design weights or states associated with noise or uncertainty models and does not explicitly model the dynamics of the flight system. Moreover, rarely is a spacecraft instrumented to directly measure the disturbances even if the disturbance sources are fully known. Since the disturbance time histories are not measured (required for system identification), $B_{1}$ is not included above in Eq. 40. If on-orbit dynamics characterization is anticipated, then excitation actuators (disturbances) may be included in the system design. But these actuators can also be used for feedback control as well and as such would be accounted for in the $B_{2}$ matrix. Hence, all measured inputs are accounted for in the $B_{2}$ matrix and the estimation of $B_{1}$ is not required. This has the beneficial effect of reducing the number of parameters to estimate and hence reducing the chromosome length.

For this example, the true plant is described by an identity mass matrix and the stiffness values are $K_{1}=25, K_{2}=12.5$, and $K_{3}=1$. These values result in an open loop system with undamped modes at $0.145 \mathrm{~Hz}, 0.347 \mathrm{~Hz}, 0.688 \mathrm{~Hz}$, and $1.156 \mathrm{~Hz}$. A noncollocated sensor/actuator configuration is adopted with the outputs consisting of the angular displacement of disks three and four while the control torque is applied to disk one.

Case A

The first case begins with an estimated model that has a $10 \%$ error in the stiffness matrix and a $5 \%$ error in the mass matrix. With eight states, one input, and two outputs, the chromosome was a string of $n *(p+m)=24$ parameters encoded as an eight bit integeter. The eight bits mapped into a range of $\pm 10 \%$ for each parameter.

An initial population of 4000 chromosomes was generated with random binary bit strings. In order to preserve the best member of each generation, an elitist strategy was implemented where the most fit $10 \%$ of the population was directly copied into the subsequent generation. Tournament selection was used with random pairing of two chromosomes; the probability of crossover was 0.75 , and binary mutation occurred for $50 \%$ of the parameters (the probability per bit was 0.0625 with eight bits per parameter). This was an atypically high probability of mutation because the objective was not to converge the entire population to the most fit member as in the typical genetic algorithm. For this application, the one most fit member was sought and a high mutation rate was employed to continually infuse new information content into the gene pool.

Feasibility of solutions was enforced by checking the closed loop stability for each member of the population and rejecting those members that rendered the closed loop unstable. Clearly the difficulty of populating 
the gene pool with feasible plants is a function of the control system. For this reason also a robust controller is most suitable for closed loop system identification (as described earlier). A low authority LQG controller was designed for the initial plant and held fixed for the plant estimation phase. In a flight application, the best plant identified during this step would be used for a controller update. This iterative identification-tuning process could be repeated until acceptable performance was achieved.

Figure 2 presents the evolution of the gene pool for 350 generations for two runs. Each run begins with the same initial erroneous closed loop plant but with different random initial populations and different disturbance time histories excited through the control actuator. The forty second disturbance input time history is a sum of four random time histories (with intensities of $0.25,0.5,0.75$, and 2). The disturbance input is band limited by a 6 th order butterworth filter with a break frequency at one-half the sample frequency of $10 \mathrm{~Hz}$.

Several different criteria can be applied to determine the suitability of an estimated plant. Fitness alone is difficult to correlate to absolute model errors because the cost is an integral of the output error squared over the duration of the time history. Nor is fitness is an exact basis for comparison between models since different time histories are used for different runs. However, because the dynamics

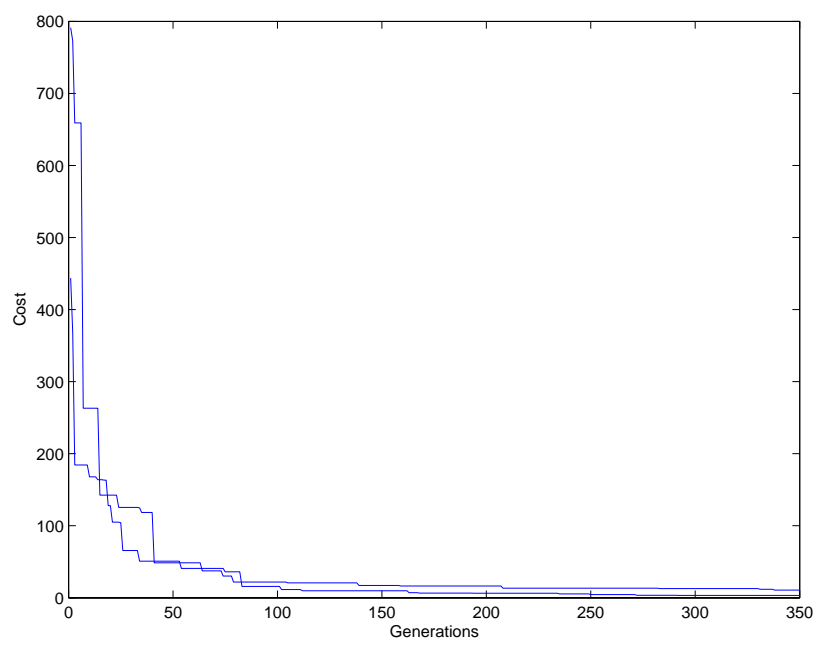

Figure 2. Fitness Evolution for Case A are realized in a canonical set of basis vectors, the parameter values themselves are equivalent for equivalent models. But determining the significance of an error in those parameters is not straightforward. Table 1 presents the parameters the true plant, the initial plant, and the best estimate for two runs (370 generations and 800 generations, respectively). The first eight rows are elements of the $A$ matrix and the final three are elements of the $B_{1}$ matrix. Note that in the first case, the best estimate in terms of fitness was achieved with larger errors in two elements of the $A$ matrix. In the second case, errors in all elements of the $A$ matrix were improved upon. This is an indirect indication that not every element of the $A$ matrix contributes equally to the cost and larger error in some parameters is more acceptable than others. Note that errors in the elements of $B_{1}$ tend to have less effect on the cost than errors in $A$.

\begin{tabular}{|c|c|c|c|c|c|c|}
\hline True & Init. & Est. 1 & Est. 2 & Init. \% error & Est. 1 \% error & Est. 2 \% error \\
\hline-625.00 & -685.94 & -639.40 & -629.72 & 9.75 & 2.30 & 0.76 \\
-62.50 & -65.48 & -62.32 & -62.37 & 4.76 & -0.29 & -0.21 \\
25.00 & 27.44 & 25.55 & 24.80 & 9.75 & 2.22 & -0.79 \\
1.00 & 1.05 & 1.09 & 1.02 & 4.76 & 8.58 & 2.26 \\
156.25 & 171.49 & 169.27 & 163.62 & 9.75 & 8.33 & 4.71 \\
156.25 & 163.69 & 168.25 & 162.73 & 4.76 & 7.68 & 4.15 \\
-12.50 & -13.72 & -12.98 & -12.66 & 9.75 & 3.85 & 1.27 \\
-14.50 & -15.19 & -14.86 & -14.59 & 4.76 & 2.50 & 0.61 \\
37.50 & 37.42 & 37.66 & 37.22 & -0.23 & 0.44 & -0.74 \\
1.00 & 0.95 & 1.01 & 1.01 & -4.76 & 1.03 & -0.39 \\
-1.00 & -1.00 & -1.01 & -0.97 & -0.23 & 0.67 & -3.24 \\
\hline
\end{tabular}

Table 1. True, Initial, and Estimated Parameters for Case A

Another means of comparison is the eigenvalues of the open loop plants. The eigenvalues have a more direct effect on the time response of the system than do the individual elements of the plant matrices and as such are a valuable basis for comparison. Figure 3 shows the very significant reduction in error associated 
with frequencies accomplished by the genetic algorithm parameter optimization. This demonstrates the not surprising fact that like the individual matrix elements, not every mode contributes equally to the cost. As with the parameter errors, second run yielded a more accurate model by this means of evaluation. Both estimates are quite accurate in terms of parameter values and open loop eigenvalues for this case.

Case B

The second case begins with an estimated model that has a $25 \%$ error in the stiffness matrix and employs a ten bits per parameter binary encoding mapped into a range of $\pm 50 \%$ for each parameter. Otherwise the parameters are the same as Case A. Table 2 presents the comparison of parameter estimates which indicates a significant reduction in error with most parameters, although some parameter errors are actually increased. Each of the four modes in the initial model erred by $9.0 \%$ while the estimate modes erred by $-1.0436 \%, 0.50 \%, .027 \%$, and $-0.38 \%$. This case clearly demonstrates how the mode frequencies of the model can very accurately match the true plant even though the errors in some evidently insignificant parameters can be quite large.

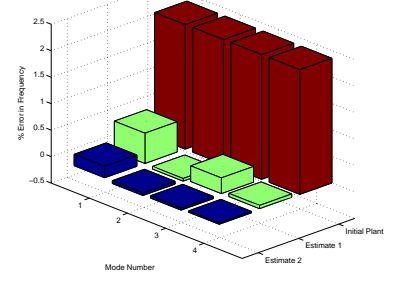

Figure 3. Error in Open Loop Plant Eigenfrequencies for Case A

\begin{tabular}{|c|c|c|c|c|}
\hline True & Init. & Est. & Init. \% error & Est. \% error \\
\hline-625.00 & -885.77 & -545.06 & 41.72 & -12.79 \\
-62.50 & -74.40 & -58.29 & 19.05 & -6.73 \\
25.00 & 35.43 & 35.07 & 41.72 & 40.27 \\
1.00 & 1.19 & 1.78 & 19.05 & 78.46 \\
156.25 & 221.44 & 136.48 & 41.72 & -12.65 \\
156.25 & 186.01 & 140.10 & 19.05 & -10.34 \\
-12.50 & -17.72 & -15.82 & 41.72 & 26.55 \\
-14.50 & -17.26 & -18.35 & 19.05 & 26.55 \\
37.50 & 42.52 & 32.98 & 13.38 & -12.06 \\
1.00 & 0.95 & 1.00 & -4.77 & -0.43 \\
-1.00 & -1.13 & -1.57 & 13.38 & 56.55 \\
\hline
\end{tabular}

Table 2. True, Initial, and Estimated Parameters for Case B

\section{Case $C$}

The final case includes a nonzero and uncertain damping. This case begins with an estimated model that has a $35 \%$ error in the stiffness matrix and $50 \%$ error in the damping ratio with the binary strings mapped into a range of $\pm 75 \%$ for each parameter. Otherwise, the parameters are the same as Case B. Table 3 presents the comparison of parameter estimates while Figure 4 presents a comparison of eigenvalues for Case B. Note that only the $A$ matrix parameters for the first two genetic algorithm runs are included while two additional runs are included in the mode frequency error bar plot. The first estimate is the most accurate of the four runs considered, each of which demonstrated considerable error in the frequency of the fourth mode. This case shows the difficulty encountered with significantly larger uncertainties in the model. However, standard local optimization routines are typically much less robust to modeling errors. ${ }^{5}$ Future research will compare the local optimization alone to the genetic algorithm/local hybrid optimization for this example.

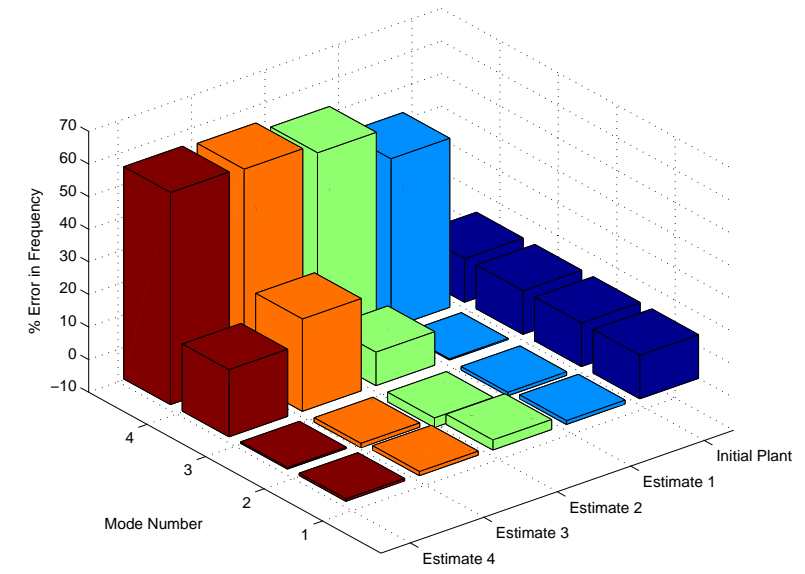

Figure 4. Error in Open Loop Plant Eigenfrequencies for Case $\mathbf{C}$ 


\begin{tabular}{|c|c|c|c|c|c|c|}
\hline True & Init. & Est. 1 & Est. 2 & Init. \% error & Est. 1 \% error & Est. 2 \% error \\
\hline-625 & -1033.2 & -882.43 & -947.57 & 65.306 & 41.189 & 51.611 \\
-68.75 & -93.112 & -138.64 & -148.2 & 35.436 & 101.66 & 115.57 \\
25 & 41.327 & 29.48 & 34.934 & 65.306 & 17.92 & 39.735 \\
1.25 & 1.7959 & 1.0125 & 1.0178 & 43.673 & -19.00 & -18.578 \\
156.25 & 258.29 & 186.14 & 233.11 & 65.306 & 19.132 & 49.188 \\
157.81 & 204.08 & 147.97 & 191.96 & 29.319 & -6.2342 & 21.64 \\
-12.5 & -20.663 & -16.497 & -21.678 & 65.306 & 31.978 & 73.426 \\
-14.625 & -18.898 & -17.609 & -22.569 & 29.217 & 20.407 & 54.321 \\
\hline
\end{tabular}

Table 3. True, Initial, and Estimated $A$ Matrix Parameters for Case C

\section{References}

\footnotetext{
${ }^{1}$ R.E. Skelton. Model error concepts in control design. International Journal of Control, 49(5):1725-1753, 1989.

${ }^{2}$ K. Liu and R.E. Skelton. Closed loop identification and iterative controller design. In Proceedings of The IEEE Conference on Decision and Control, 1990.

${ }^{3}$ M. Phan, J.N. Juang, L.G. Horta, and R.W. Longman. System identification from closed-loop data with known output feedback dynamics. Journal of Guidance, Control, and Dynamics, 17(4):661-669, 1994.

${ }^{4}$ L. Ljung. System Identification:Theory for the User. Prentice-Hall, Inc., Englewood Cliffs, NJ, 1987.

${ }^{5}$ M.S. Whorton and A. J. Calise. On-orbit model refinement for controller redesign. In Proceedings of The IEEE Aerospace Conference, 1998.

${ }^{6}$ R.J.P. Schrama. Accurate identification for control: The necessity of an iterative scheme. IEEE Transactions on Automatic Control, 37(7):991-994, July 1992.

${ }^{7}$ T. Kailath. Linear Systems. Prentice-Hall, Inc., 1980.

${ }^{8}$ L. Mitchell. An Introduction to Genetic Algorithms. The MIT Press, Massachusetts Institute of Technology, Cambridge, MA $02142,1998$.

${ }^{9}$ R.H. Cannon Jr. and D.E. Rosenthal. Experiments in control of flexible structures with noncolocated sensors and actuators. Journal of Guidance, Control, and Dynamics, 7(5):546-553, 1984.
} 